\title{
COVID-19 in a patient treated with eculizumab for aquaporin-4 neuromyelitis optica
}

\author{
Ana Maria Cabal-Herrera ${ }^{1} \cdot$ Farrah J. Mateen ${ }^{1} \mathbb{C}$
}

Received: 1 April 2021 / Revised: 20 April 2021 / Accepted: 21 April 2021 / Published online: 27 April 2021

(c) Springer-Verlag GmbH Germany, part of Springer Nature 2021

\section{Dear Sirs,}

Eculizumab is an anti-C5 humanized monoclonal antibody that is approved for the treatment of aquaporin-4 (AQP-4) seropositive neuromyelitis optica (NMO). There are limited data on best treatment practices for patients with NMO, treated with eculizumab in the setting of COVID-19. We report a woman with NMO and multiple comorbidities who presented with SARS-CoV-2 infection, days after infusion with eculizumab, experiencing a favorable outcome while continuing eculizumab.

A 56-year-old woman with AQP-4 seropositive NMO, diagnosed 8 years earlier, was seen in December 2020. Concomitant diagnoses included systemic lupus erythematous (SLE) with pericarditis, positive lupus anticoagulant with deep venous thrombosis, Sjögren's disease, and myositis. Her neurologic examination was remarkable for no light perception OD, right relative afferent pupillary defect, diffuse hyporeflexia, and lower extremity monoplegia. Findings were stable compared to her prior visit (2019). Her most recent MRI brain and spinal cord had chronic white matter demyelinating changes.

She began eculizumab in February 2020 to treat her NMO and possibly NMO-related myositis. She received $900 \mathrm{mg}$ IV infusions which were interrupted due to the COVID-19 pandemic in March, after just two doses. She was managed with prednisone $7.5 \mathrm{mg}$ by mouth daily.

Eculizumab $900 \mathrm{mg}$ IV was resumed in December 2020 (Fig. 1). A few days after her first dose, a young household family member was exposed to COVID-19. Within 3 days, she presented with shortness of breath, headache, fever, and cough. These symptoms resolved after 3 days. She had a positive nasopharyngeal swab test for SARS-CoV-2 infection by reverse-transcriptase-polymerase-chain-reaction (RT-PCR) assay, 10 days after her eculizumab infusion. She did not receive any specific therapy or oxygen for COVID19 but she self-dosed her steroids, taking $20 \mathrm{mg}$ daily once then $10 \mathrm{mg}$ daily afterwards. 3 weeks later, she emergently presented for chest pain and palpitations. On physical examination, she had normal vital signs and her laboratory tests were reassuring: serum D-dimer was negative and her troponin levels were normal. Follow-up has been uneventful at 8 weeks. She continued steroids ( $7.5 \mathrm{mg}$ daily), and received eculizumab $900 \mathrm{mg}$ IV 3 and 7 weeks after her COVID-19 diagnosis. Serum COVID-19 nucleocapsid antibody was positive 49 days after her first COVID-19 symptoms.

We present a patient with PCR-confirmed, symptomatic COVID-19 while treated with eculizumab for NMO, highlighting her overall favorable outcome. There are reports of patients with other autoimmune diseases on chronic treatment with eculizumab presenting with confirmed confirmed SARS-CoV-2 infection [1-6] (Table 1); most had a mild disease course without recognized sequelae. It is possible that eculizumab renders people susceptible to SARS-CoV-2 infection; however, a protective effect against the development of severe COVID-19 disease may also occur [7-9]. Although eculizumab has been given safely during COVID19, a survey of 192 neurologists from the United States and Canada in April 2020 found that many felt eculizumab could put their patients at risk for COVID-19 [10].

Farrah J. Mateen

fmateen@mgh.harvard.edu

Ana Maria Cabal-Herrera

acabalherrera@mgh.harvard.edu

1 Department of Neurology, Massachusetts General Hospital, 165 Cambridge St., Office 627, Boston, MA 02114, USA 


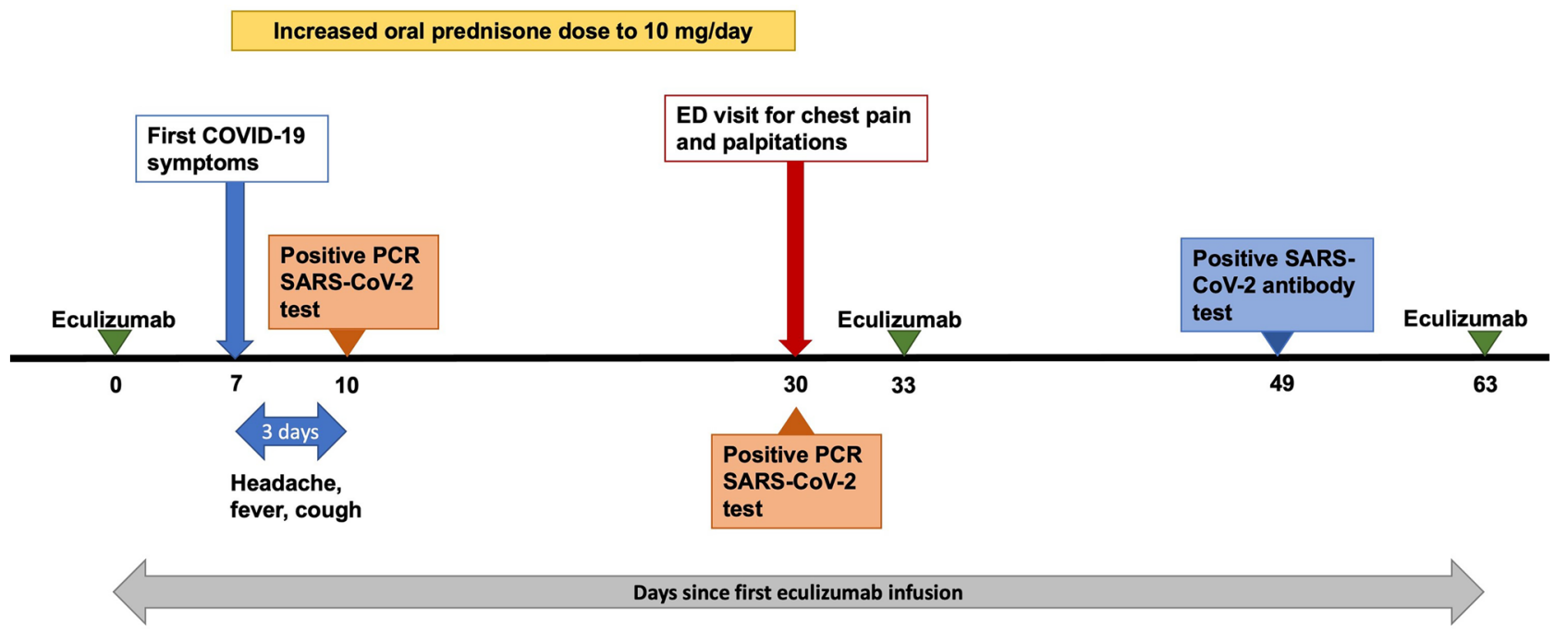

Fig. 1 Clinical course. Symptoms started 7 days after the eculizumab infusion, were mild and lasted for 3 days. The patient visited the emergency department for chest pain and palpitations, and a followup PCR test for SARS-CoV-2 was positive. The next scheduled ecu-

There is no consensus on the safe administration of eculizumab in the context of COVID-19, although recommendations exist [11]. Extrapolating from other autoimmune diseases treated chronically with eculizumab, there is no evidence to prompt its suspension in the context of a SARS-CoV-2 infection [12]. Considering the debilitating and severe nature of $\mathrm{NMO}$, it is important to balance the benefits of preventing a relapse with the potential risk of rendering a patient more susceptible to SARS-CoV-2 infection.

We also demonstrate that antibody formation to SARSCoV-2 occurs following PCR-confirmed COVID-19 in a lizumab infusions were administered without new or recurring symptoms. She had positive SARS-CoV-2 antibody test 49 days after the first eculizumab infusion. ED emergency department, $P C R$ polymerase chain reaction

patient treated with eculizumab, implying immunity to SARS-CoV-2 could occur during ongoing complement inhibitor therapy. Despite ongoing treatment with eculizumab, this patient's immune system was able to mount an antibody response to COVID-19. Antibody formation implies an immunogenic vaccine response in eculizumabtreated patients will likely occur. Meanwhile, we recommend that patients with NMO continue adopting all preventive measures against COVID-19, including vaccination, and those on eculizumab treatment should not suspend or discontinue it if exposed to SARS-CoV-2. 


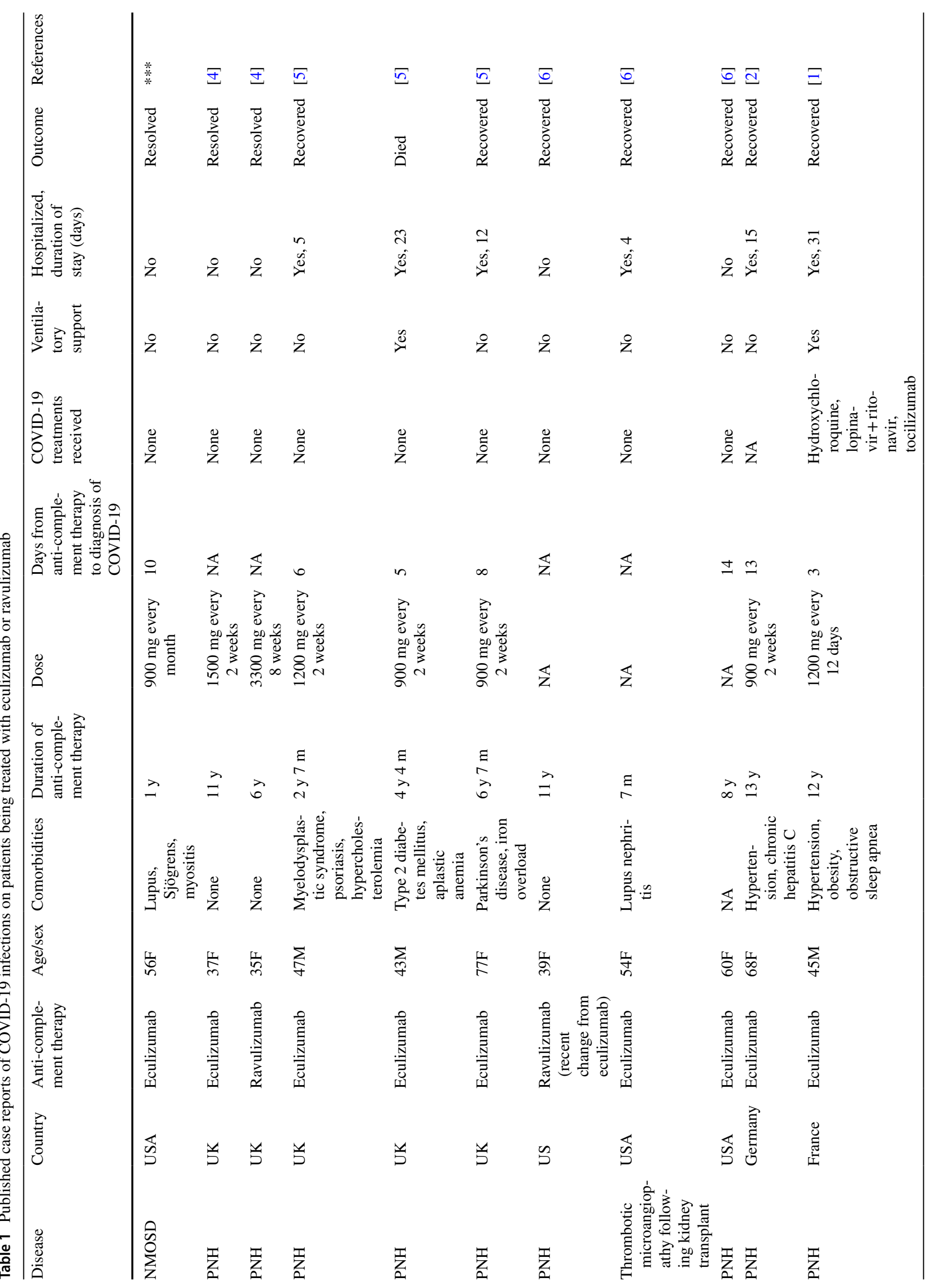




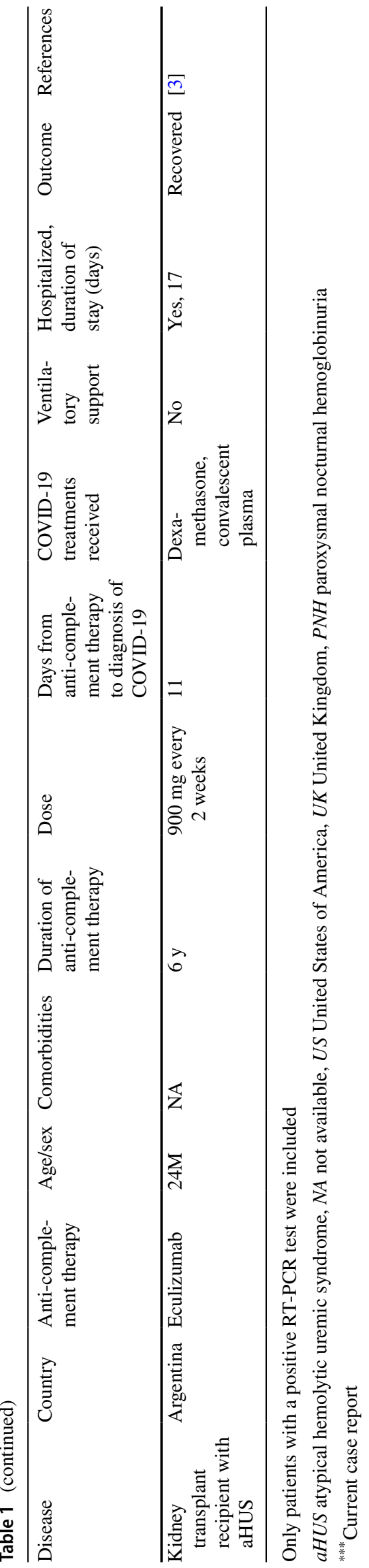

Funding The authors received no financial support for the research, authorship, and/or publication of this article.

\section{Declarations}

Conflicts of interest The authors declared no potential conflicts of interest with respect to the research, authorship, and/or publication of this article.

Informed consent Informed consent was obtained from the patient included in this study.

\section{References}

1. Genthon A, Chiarabini T, Baylac P et al (2021) Severe COVID19 infection in a patient with paroxysmal nocturnal hemoglobinuria on eculizumab therapy. Leuk Lymphoma. https://doi.org/ 10.1080/10428194.2020.1869963

2. Schüller H, Klein F, Lübbert M, Prager EP (2020) Hemolytic crisis in a patient treated with eculizumab for paroxysmal nocturnal hemoglobinuria possibly triggered by SARS-CoV-2 (COVID-19): a case report. Ann Hematol. https://doi.org/10. 1007/s00277-020-04318-6

3. Trimarchi H, Gianserra R, Lampo M et al (2020) Eculizumab, SARS-CoV-2 and atypical hemolytic uremic syndrome. Clin Kidney J 13:739-741. https://doi.org/10.1093/ckj/sfaa166

4. Kulasekararaj AG, Lazana I, Large J et al (2020) Terminal complement inhibition dampens the inflammation during COVID19. Br J Haematol. https://doi.org/10.1111/bjh.16916

5. Pike A, Muus P, Munir T et al (2020) COVID-19 infection in patients on anti-complement therapy: The Leeds National Paroxysmal Nocturnal Haemoglobinuria service experience. Br J Haematol. https://doi.org/10.1111/bjh.17097

6. Araten DJ, Belmont HM, Schaefer-Cutillo J et al (2020) Mild clinical course of COVID-19 in 3 patients receiving therapeutic monoclonal antibodies targeting c 5 complement for hematologic disorders. Am J Case Rep 21:e927418. https://doi.org/10.12659/ AJCR.927418

7. Gralinski LE, Sheahan TP, Morrison TE et al (2018) Complement activation contributes to severe acute respiratory syndrome coronavirus pathogenesis. MBio. https://doi.org/10.1128/ mBio.01753-18

8. Magro C, Mulvey JJ, Berlin D et al (2020) Complement associated microvascular injury and thrombosis in the pathogenesis of severe COVID-19 infection: a report of five cases. Transl Res 220:1-13. https://doi.org/10.1016/j.trsl.2020.04.007

9. Carvelli J, Demaria O, Vély F et al (2020) Association of COVID-19 inflammation with activation of the C5aC5aR1 axis. Nature 588:146-150. https://doi.org/10.1038/ s41586-020-2600-6

10. Rezaei SJ, Vogel AC, Gazdag B et al (2020) Neuromyelitis optica practice and prescribing changes in the setting of COVID-19: a survey of neurologists. J Neuroimmunol 346:577320. https://doi.org/10.1016/j.jneuroim.2020.577320

11. Hamdy SM, Abdel-Naseer M, Shehata HS et al (2020) Management strategies of patients with neuromyelitis optica spectrum disorder during the COVID-19 pandemic era. Ther Clin Risk Manag 16:759-767. https://doi.org/10.2147/TCRM.S261753

12. Zrzavy T, Wimmer I, Rommer PS, Berger T (2020) Immunology of COVID-19 and disease-modifying therapies: the good, the bad and the unknown. Eur J Neurol. https://doi.org/10.1111/ene.14578 